\title{
Insights into p53 transcriptional function via genome- wide chromatin occupancy and gene expression analysis
}

\author{
F Nikulenkov ${ }^{1}$, C Spinnler ${ }^{1,6}$, H Li $^{1,6}$, C Tonelli ${ }^{1}$, Y Shi ${ }^{1}$, M Turunen ${ }^{2,3}$, T Kivioja $^{2,3}$, I Ignatiev ${ }^{1}$, A Kel ${ }^{4,5}$, J Taipale ${ }^{2,3}$ and G Selivanova ${ }^{\star, 1}$
}

The tumor-suppressor p53 can induce various biological responses. Yet, it is not clear whether it is p53 in vivo promoter selectivity that triggers different transcription programs leading to different outcomes. Our analysis of genome-wide chromatin occupancy by p53 using chromatin immunoprecipitation (ChIP)-seq revealed 'p53 default program', that is, the pattern of major p53-bound sites that is similar upon p53 activation by nutlin3a, reactivation of p53 and induction of tumor cell apoptosis (RITA) or 5 -fluorouracil in breast cancer cells, despite different biological outcomes. Parallel analysis of gene expression allowed identification of 280 novel p53 target genes, including p53-repressed AURKA. We identified Sp1 as one of the p53 modulators, which confer specificity to p53-mediated transcriptional response upon RITA. Further, we found that STAT3 antagonizes p53-mediated repression of a subset of genes, including AURKA.

Cell Death and Differentiation (2012) 19, 1992-2002; doi:10.1038/cdd.2012.89; published online 13 July 2012

Given the high tumor-suppressor potency of the p53 transcription factor, the strategy to reestablish p53 function is very attractive. ${ }^{1}$ However, there remains much to learn, as the cellular response to $\mathrm{p} 53$ can range from cell death to cell survival, and the consequences of p53 reactivation are

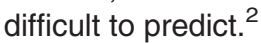

Several classes of molecules reactivating p53, such as nutlin3a, reactivation of p53 and induction of tumor cell apoptosis (RITA), PRIMA-1 ${ }^{\mathrm{MET}} / \mathrm{APR}-246, \mathrm{MI}-219$ and tenovins, have been shown to efficiently eliminate tumor cells in vitro and in animal models. ${ }^{3-7}$ Nutlin3a binds the major p53 inhibitor Mdm2, entailing the induction of $\mathrm{p} 53 .^{3}$ In contrast, RITA prevents Mdm2 binding and activates p53 by binding to p53 itself. ${ }^{4}$ Notably, p53 activation by nutlin3a and RITA in the same cancer cells triggers different transcriptional programs, resulting in growth arrest or apoptosis. ${ }^{8}$

The control of cell fate by p53 is accomplished by activation or repression of p53-target genes, containing the consensus p53-binding motif. ${ }^{9}$ However, distinct transcriptional programs triggered by $\mathrm{p} 53$ upon different stresses raise the question whether this is due to a differential pattern of p53 binding to promoters, or whether p53 binds the same set of its targets irrespective of the type of stimuli, while the presence or absence of other factors will decide whether the expression of a particular gene will be regulated by p53. ${ }^{10}$ Genome-wide analyses are required to delve into the mechanisms by which it triggers different transcriptional programs in different settings. Previous genome-wide studies of p53 DNA binding in vivo using chromatin immunoprecipitation (ChIP) in conjunction with hybridization (ChIP-chip) or ChIP paired-end (ChIP-PET) sequencing approaches helped to refine the p53 consensus binding site and to predict a number of p53-target genes. $^{11-13}$

In this study, we carried out an unbiased analysis of chromatin occupancy by p53 using p53 ChIP followed by deep sequencing (ChIP-seq) upon its induction by three different molecules, target-specific nutlin $3 a$ and RITA, as well as chemotherapeutic drug 5-fluorouracil (5-FU) and combined it with the analysis of a new comprehensive data set of p53-induced gene expression changes. We characterized important features of p53 in vivo chromatin occupancy and identified novel target genes and transcription factors, which affect p53 transcriptional function, as exemplified by the modulation of p53 transcriptional response by Sp1 and the antagonistic interaction between p53 and STAT3 at the AURKA promoter.

\footnotetext{
${ }^{1}$ Department of Microbiology, Tumor and Cell Biology (MTC), Karolinska Institutet, Stockholm, Sweden; ${ }^{2}$ Department of Medical Genetics, Genome-Scale Biology Research Program, Biomedicum Helsinki, University of Helsinki, Helsinki, Finland; ${ }^{3}$ Department of Bioscience and Nutrition, Karolinska Institutet, Stockholm, Sweden; ${ }^{4}$ geneXplain GmbH, Wolfenbüttel, Germany and ${ }^{5}$ Institute of Chemical Biology and Fundamental Medicine, Russian Academy of Sciences, Novosibirsk, Russia

*Corresponding author: G Selivanova, Department of Microbiology, Tumor and Cell Biology (MTC), Karolinska Institutet, Nobels väg 16, Box 280, SE-17177 Stockholm, Sweden. Tel: +46 8 52486302; Fax: +46 8 330744; E-mail: Galina.Selivanova@ ki.se

${ }^{6}$ These authors contributed equally to this work.

Keywords: p53; ChIP-seq; repression; Sp1; STAT3; microarray

Abbreviations: RITA, reactivation of p53 and induction of tumor cell apoptosis; 5-FU, 5-fluorouracil; qPCR, quantitative real-time reverse transcription PCR; stattic, STAT three inhibitory compound; ChIP, chromatin immunoprecipitation; ChIP-seq, ChIP combined with deep sequencing; ChIP-PET, ChIP paired-end; ChIP-chip, ChIP in conjunction with chip hybridization; ChIP-PCR, ChIP combined with real-time reverse transcription PCR; TSS, transcription start site; TF, transcription factor; $\mathrm{RE}$, response element

Received 19.12.11; revised 11.6.12; accepted 11.6.12; Edited by M Oren; published online 13.7.12
} 


\section{Results}

Genome-wide map of p53 binding to chromatin upon its induction by nutlin3a, RITA and 5-FU. We compared the genome-wide chromatin occupancy by p53 upon its activation with three different compounds, non-genotoxic molecules nutlin3a and RITA, as well as chemotherapeutic drug 5-FU, known to induce DNA damage. ${ }^{14}$ Despite mechanistic differences, all the three treatments induce p53 protein levels and its transcriptional activity. ${ }^{3,8,15}$ Intriguingly, they lead to different biological responses: depletion of S-phase indicating growth arrest upon nutlin3a treatment, mainly G1/S arrest induced by 5-FU and induction of apoptosis as a major response to RITA (Figure 1a).

We performed the p53 ChIP followed by massive parallel DNA sequencing (ChIP-seq) in MCF7 cells treated with nutlin3a, RITA and 5-FU. Applying a threshold of $P<0.05$, we identified 6357 peaks corresponding to p53-bound DNA fragments in the library from mock-treated control cells, and 17882 in nutlin3a-, 11412 in RITA- and 11257 in 5-FU-treated samples (Figure 1b, Supplementary Tables S1-4).
Comparison of the chromatin occupancy by p53 upon different treatments using the web-based tool Galaxy Genome ${ }^{16}$ revealed a partial overlap between the data sets (Figure 1b). In order to select the most relevant binding sites, we selected peaks with a minimum of two-fold enrichment over IgG control and large area, which correlated with peak height in a linear fashion (Supplementary Figure S1A). The increase of stringency (area 30 ) resulted in a significant increase of the proportion of peaks common to all three treatments, for example, for nutlin $3 a$, the proportion of common peaks increased from 20 to $78 \%$ (Figure 1b). These results indicate that p53 binds the same set of sites, defined as a 'default p53 program' independent from the type of p53 activating stimuli.

Our ChIP combined with real-time reverse transcription PCR (ChIP-PCR) analysis confirmed p53 binding only to a half of randomly selected sites from 'area 10' (data not shown), suggesting that these are low confidence peaks. Therefore, for further analysis, we selected all p53-bound loci, which reached the threshold area 20 . For these high confidence peaks, the proportion of those common for all
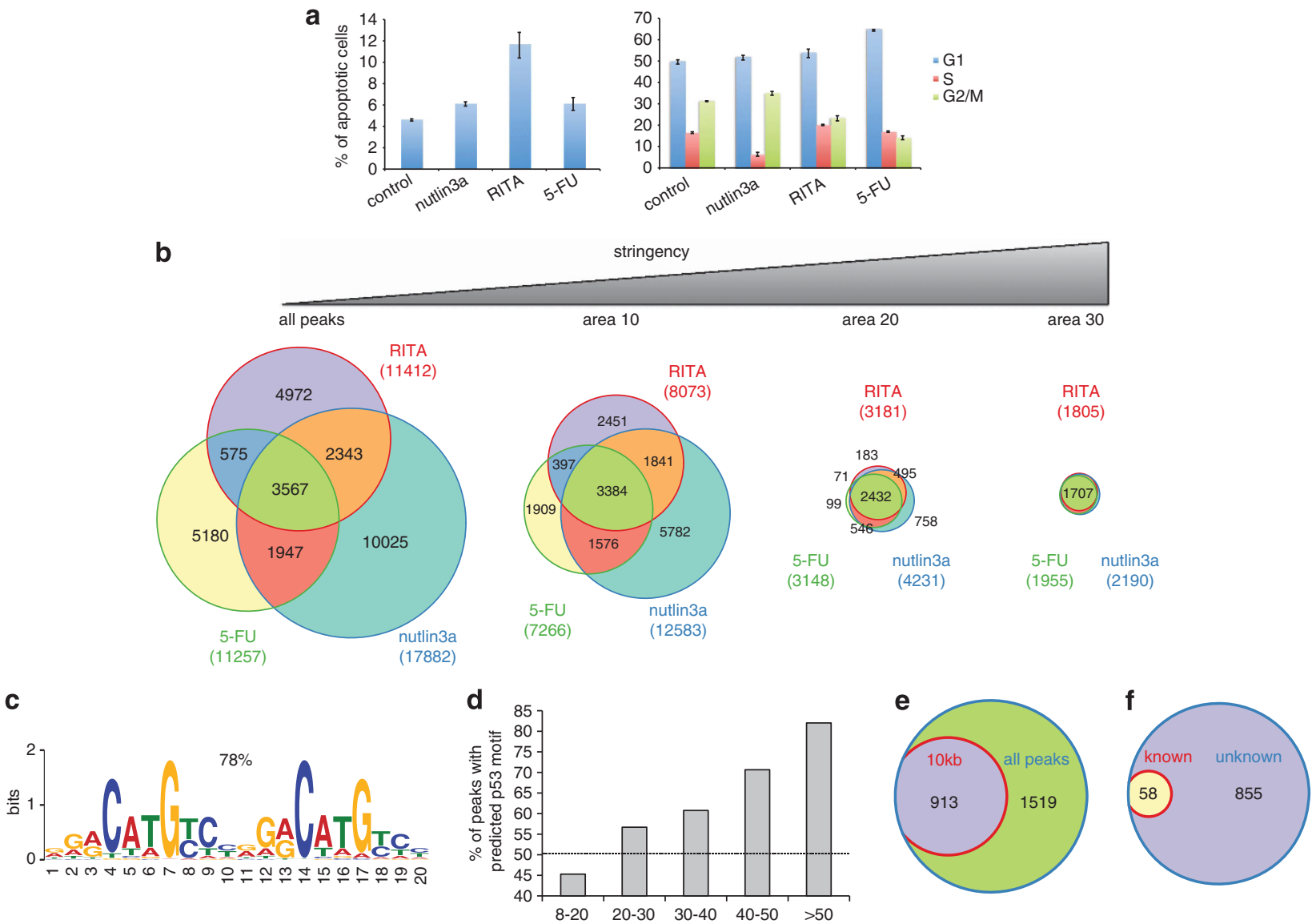

Figure 1 Characterization of the global chromatin occupancy by p53 upon its activation by nutlin3a, RITA and 5-FU. (a) Induction of apoptosis upon nutlin3a, RITA and 5-FU treatment of MCF7 cells was detected by fluorescence-activated cell sorting (FACS) of annexin V-stained cells (left) and cell-cycle profiles were assessed by FACS of $\mathrm{Pl}$-stained cells (right). (b) Venn diagrams were obtained by intersection of all p53-bound DNA fragments (ChIP-Seq peaks, $P<0.05$ ) obtained from MCF7 cells treated with nutlin3a-, 5-FU- and RITA and filtering according to the area. (c) The p53 consensus binding motif was identified de novo by analyzing the sequences of 500 randomly selected peaks common for all the three treatments using the program MEME (d). The fraction of peaks containing the $\mathrm{p} 53$ consensus site increased along with increased stringency of peak selection. (e) A Venn diagram demonstrates the proportion of p53-bound fragments located within $\pm 10 \mathrm{~kb}$ of the TSS (913 out of 2432). (f) A Venn diagram shows the proportion of peaks located in the vicinity of known and unknown p53-target genes bound within $\pm 10 \mathrm{~kb}$ of TSS upon all three treatments. See also Supplementary Figure S1, Supplementary Figure S2 and Supplementary Tables S1-S4 
the three treatments (a total of 2432 peaks) became predominant, although we detected a certain number of unique peaks (area 20, Figure 1b). However, ChIP-PCR analysis did not confirm their specificity: no unique peaks were found among randomly selected top-score nutlin3a-only peaks, whereas merely $40 \%$ of RITA-only peaks were confirmed as being specific (data not shown). Moreover, microarray analysis showed that the expression of the majority of genes, which appear to be selectively bound and significantly changed upon RITA treatment, was also affected by nutlin3a (24 out of 30 genes, Supplemental Table 12). In addition, as indicated in Supplementary Figure S1B, only $10 \%$ of peaks detected upon nutlin3a and less than a half of RITA-specific peaks contain p53 consensus motif. In contrast, $82 \%$ of peaks detected upon all three conditions (i.e., common peaks) contain p53 consensus.

Thus, we conclude that the majority of high-confidence binding sites are common for all the three treatments and represent the 'p53 default program'.

In vivo p53-binding sequence preference. We generated a consensus p53-binding site based on p53-bound fragments common for all the three treatments at threshold area 20 (Figure 1b) by applying the de novo motif discovery algorithm MEME for 500 randomly selected common peaks (out of $2432, E=4.4 \times 10^{-682}$ ) (Figure 1c). This motif fits well to the original p53 consensus site as well as the one refined recently. ${ }^{11,12,17,18}$ Interestingly, applying a higher threshold for the peak area increased the proportion of peaks containing the p53-binding site from 45 to $82 \%$, as detected by the p53Scan algorithm (Figure 1d), whereas only $3.9 \%$ of random sequences contain this motif. Thus, most of the 'default p53 program' sites contained canonical p53-binding sites.

In agreement with previous report, ${ }^{12}$ we found that $37 \%$ of the peaks are located within $10 \mathrm{~kb}$ from the transcription start sites (TSSs, Figure 1e and Supplementary Figure S2).

Identification of novel p53-target genes. Analysis of p53 occupancy at promoters showed that, along with a number of known p53 targets, p53 bound to 855 sites corresponding to 688 novel genes (Figure 1f). Comparison of gene-expression profiles with our ChIP-seq data showed that $41 \%$ of these (280 genes) were differently expressed upon nultin3a (Figures $2 a$ and $b$, see Supplemental Data and Supplementary Tables S5-S9 for a more detailed description). These genes are likely to be novel functional p53-target genes (Supplementary Table S10).

A total of 4484 genes that changed their expression upon nutlin3a, but were not bound by p53 in the vicinity of TSS, most likely represent the secondary effects of p53. In addition, we detected the p53 binding to the promoters of 408 genes, which did not result in expression changes (Figure $2 a$ and Supplementary Tables S5-S9). Thus, our data suggest that the binding of p53 per se is not sufficient to regulate gene expression.

Comparison of p53-bound and induced versus p53-bound and repressed genes. Among 280 novel p53-target genes, 214 were induced and 66 were repressed (Figure 2b).
Using gene ontology, we functionally annotated these genes (Figure 2c, Supplementary Table S10). Along with expected categories, such as apoptosis, cell-cycle regulation, DNA repair/damage, signal transduction, metabolism, transport/ ion channels, protein catabolism and translation/splicing, we found that one of the most highly represented categories involved genes encoding regulators of transcription, mainly transcription factors (TFs) (e.g., E2F7, HES1, HES2, FOSL1, ZNF219, Sp1). This might help to explain the gross changes in gene expression upon p53 activation, that is, the differential expression of 4484 genes after nutlin3a treatment.

We searched for the p53-binding motif in our set of p53-target genes and applied the de novo discovery algorithm MEME to the p53-bound fragments separately for induced and repressed genes, which resulted in two distinct motifs, both of which are similar to the p53 consensus motif. However, although this motif was present in $66 \%$ of induced genes (E-value of $10^{-244}$ ), the proportion of repressed genes, which contained the p53 consensus was much lower, only $21 \%$ (E-value reached $5.3 \times 10^{1}$ ) (Figure $2 d$ ). This conclusion was confirmed using p53Scan and p53MH programs (Figure $2 \mathrm{e}$ and Supplementary Figure S5B). Interestingly, the extent of occupation by p53 was significantly higher in induced than in repressed genes (Supplementary Figure S5A).

Taken together, our data implies that the majority of p53-bound genes, which are induced, share the classical consensus motif and have more pronounced p53 binding than the repressed genes. The consensus motif generated from repressed targets (Figure $2 \mathrm{~d}$, lower panel) fits with the classical consensus site even better, but is infrequently present. Interestingly, analysis of p53 occupancy in control and treated samples allowed to identify two classes of genes bound by p53 in the absence of activating stimuli (Supplementary Figures S3 and S4).

Analysis of composite response elements (REs) in the promoters of p53-repressed genes revealed two possible modes of repression by p53. We performed composite pattern search for TF-binding sites adjacent to sites occupied by p53 (distance $<100 \mathrm{bp}$ ) in the promoters of repressed genes using geneXplain platform. ${ }^{19}$ Top-score TF include E2F, MYB, HNF3, DEAF-1, HSF-2, IRF, STAT and others (Table 1). E2F1 is known to enhance apoptosis induced by p53 and cooperate with p53 in activation of several genes; moreover, E2F and p53 have been shown to repress two anti-apoptotic genes $\mathrm{BCl}-2$ and $\mathrm{Mcl}-1 .{ }^{20}$ Further, E2F1 is activated by nutlin3a and its transcriptional activity is a crucial determinant of suppressor effect of nutlin3a. ${ }^{21}$ Enrichment of E2F RE next to p53-binding sites in repressed genes suggests that the cooperation between these two factors in transcriptional repression via direct binding of both TFs to promoters might occur on a larger scale than previously anticipated.

Apart from acting together in induction of gene expression, IRF and p53 have been shown to cooperate in repression of hTERT gene. ${ }^{22}$ Taken together with our data, this provides a support to the notion that p53 and IRF can collaborate in repression of at least several genes. 
a

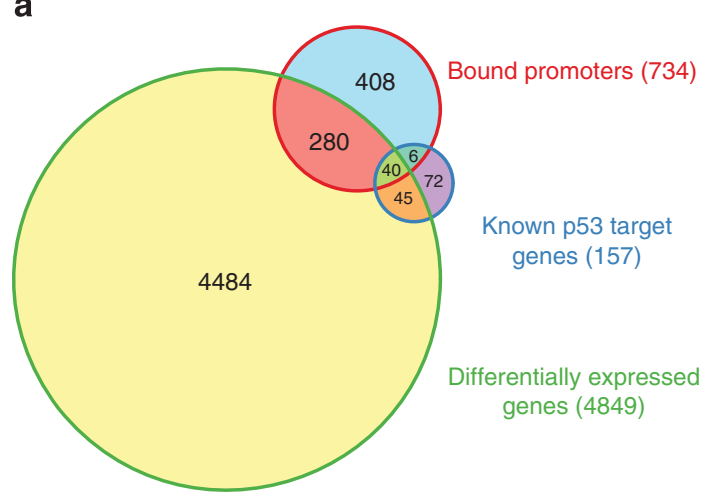

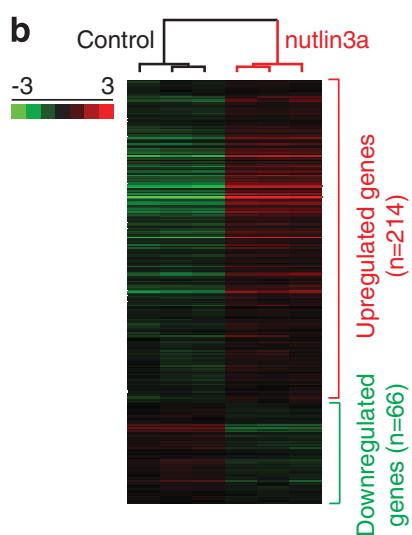

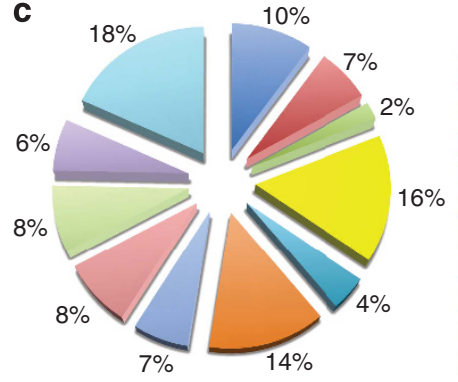

d
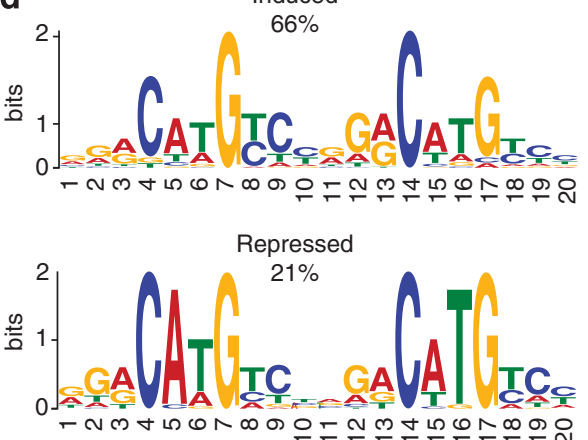

Apoptosis/Proliferation

DNA damage/DNA repair

Differentiation/Development

Transcription

Protein Catabolism

Signal Transduction

Cell Adhesion/Mobility/Angiogenesis

Metabolism

Transport/lon Channels

Translation/RNA Splicing

Unknown/Other

\section{e}

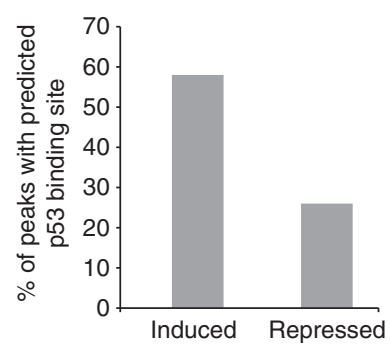

Figure 2 Novel p53-target genes bound by p53 and differentially expressed upon nultin3a treatment. (a) Bioinformatic analysis of the genes bound by p53 within $10 \mathrm{kbp}$ from TSS and differentially expressed upon $8 \mathrm{~h}$ of nutlin3a treatment $(P<0.05)$ revealed 280 putative novel p53-target genes. (b) A heatmap shows the differential expression of selected 280 p53-bound genes upon nutlin3a treatment. Columns indicate separate arrays and rows indicate genes. The values are row-mean-centered. Green corresponds to lower and red to higher than mean value expression. (c) The pie chart of the functional categories of 280 novel p53-target genes. (d) The p53 consensus binding site was detected much more often in the proximity of TSS of induced, than repressed genes (66\% and $21 \%$, respectively), as analyzed using the MEME algorithm. (e) The probability of the presence of a p53 consensus binding motif in the p53-bound fragment is higher in induced than in repressed genes

Table 1 Composite regulatory elements in p53-repressed promoters

\begin{tabular}{lcccc}
\hline $\begin{array}{l}\text { Composite } \\
\text { elements }\end{array}$ & $\begin{array}{c}\text { Frequency } \\
\text { in peaks } \\
\text { (fP) }\end{array}$ & $\begin{array}{c}\text { Frequency in } \\
\text { background } \\
\text { (fB) }\end{array}$ & Ratio & $P$-value \\
& \multicolumn{1}{c}{ (fB) } & & \\
\hline P53-E2F & 0.23849 & 0.029404 & 8.1108 & $5.9188 \mathrm{E}-09$ \\
P53-MYB & 0.29784 & 0.046216 & 6.44452 & $3.9883 \mathrm{E}-07$ \\
P53-GATA4 & 0.790771 & 0.588456 & 1.34381 & $2.3550 \mathrm{E}-02$ \\
P53-HNF3 & 0.304779 & 0.101922 & 2.99032 & $1.5959 \mathrm{E}-04$ \\
P53-DEAF-1 & 0.48582 & 0.179621 & 2.70469 & $5.5473 \mathrm{E}-06$ \\
P53-HSF-2 & 0.310844 & 0.085733 & 3.62572 & $1.1939 \mathrm{E}-05$ \\
P53-STAT & 0.239011 & 0.024201 & 9.87608 & $7.9175 \mathrm{E}-09$ \\
P53-IRF & 0.093085 & 0.00199 & 46.7764 & $3.6570 \mathrm{E}-06$ \\
P53-TBX5 & 0.123387 & 0.005495 & 22.4544 & $7.5897 \mathrm{E}-07$ \\
\hline
\end{tabular}

Several top-score TF, which have their RE in p53repressed genes are proto-oncogenes, including MYB, HNF3 (FOXA), DEAF-1, HSF-2 and STAT (Table 1). It is tempting to speculate that p53 might mediate transrepression via inhibition of these TF at their target promoters. Indeed, it has been found that p53 can antagonize MYB by binding to MYB-activated promoter ${ }^{23}$ and to compete out HNF3(FOXA) from its promoters. ${ }^{24}$ We addressed the question whether there is an antagonistic interaction between p53 and STAT3 at the promoters in a series of experiments presented below.

Thus, analysis of TF RE overrepresented in p53-bound and repressed promoters revealed two possible modes of p53-mediated repression: via cooperation with factors, such 
a ChIP-qPCR - chromatin occupancy by p53 (MCF7)

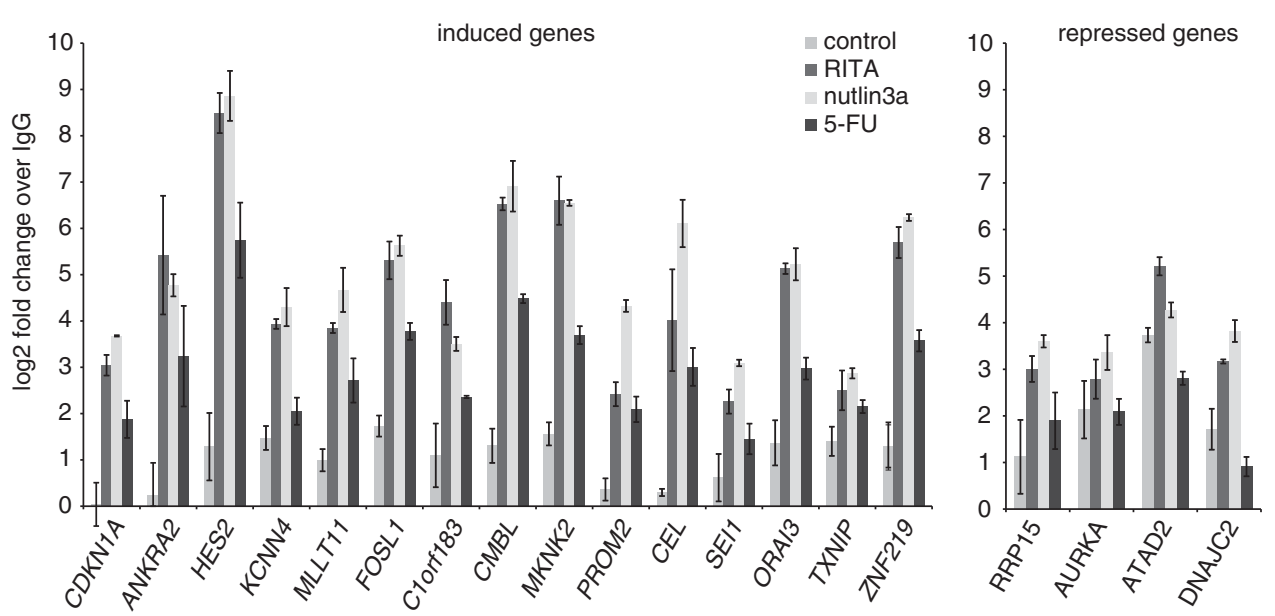

\section{b $\quad$ RT-qPCR - gene expression levels (MCF7)}
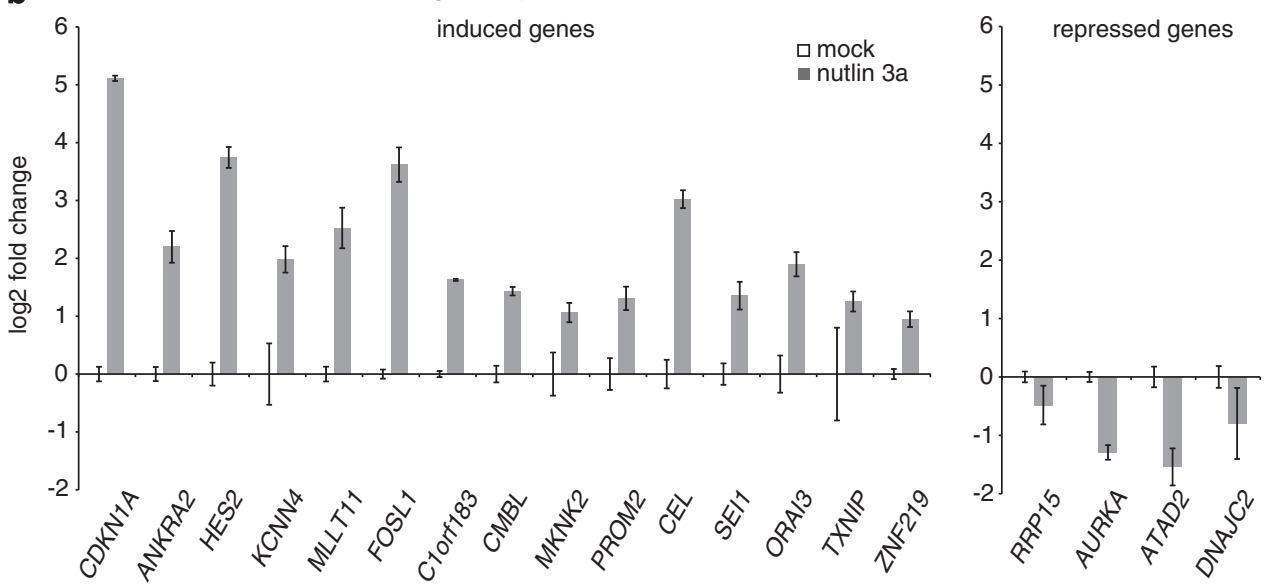

Figure 3 Validation of chromatin occupancy and mRNA expression of a selected panel of newly identified p53-target genes. (a) ChIP using the anti-p53 antibody DO-1, followed by qPCR, was used to detect relative p53 occupancy in the promoters of 18 selected genes in MCF7 cells treated with nutlin3a, RITA and 5-FU for $8 \mathrm{~h}$. CDKN1A serves as a positive control for p53 binding. (b) The mRNA levels of 18 selected genes were detected by qPCR in MCF7 cells treated with nutlin3a

as E2F and IRF, and inhibition of activity of proto-oncogenes, such as MYB, HNF3 and STAT, at their target promoters.

Validation of a set of novel p53-target genes. Assessment of $p 53$ binding to a set of 14 activated and 4 repressed novel p53 targets by ChIP-PCR, as well as their differential expression using quantitative PCR (qPCR), both in MCF7 and HCT116 cells, confirmed that the selected genes are novel p53-target genes (Figures $3 a$ and $b$ and Figure $4 a$, respectively). In addition, ectopic expression of p53 in the p53-null HCT116 TP53- / - led to the induction of 10 out of 14 genes (Figure 4c).

One of the most interesting novel p53 targets is AURKA, encoding aurora kinase $A$, a promising target for the development of novel anticancer therapies. ${ }^{25}$ In line with mRNA decline as detected by qPCR (Figures 3b and 4a), the level of aurora kinase A protein was strongly decreased in MCF7 and HCT116 cells upon p53 induction by nutlin3a, RITA and 5-FU (Figure 4b).
A more detailed investigation of the role of the p53-binding site in the promoters of $S E / 1$, as a representative of a p53induced gene, and $A U R K A$, representing a p53-repressed gene, using luciferase reporter assay confirmed the functionality of p53 RE in these genes (Figures $4 d$ and e). Taken together, our results provide a solid evidence that $A U R K A$ is directly repressed by $\mathrm{p} 53$, whereas $S E / 1$ is induced by $\mathrm{p} 53$.

Transcription-factor-binding motif enrichment in p53-target genes. Our finding of the p53 default program implies that differential gene expression upon p53 binding to its promoters is highly dependent on the presence of other TFs at p53-target promoters. In order to identify these factors, we searched for TFs, which have their binding motifs in the interval of $\pm 100 \mathrm{bp}$ of the ChIP-seq peak maximum in p53-induced and -repressed genes. Using the Match algorithm from geneXplain, we found that RE for a number of TFs, such as Sp1, WT1, ZF5, ETS1 and AP2, as well as p53, were overrepresented in the promoters of both categories of genes 
a

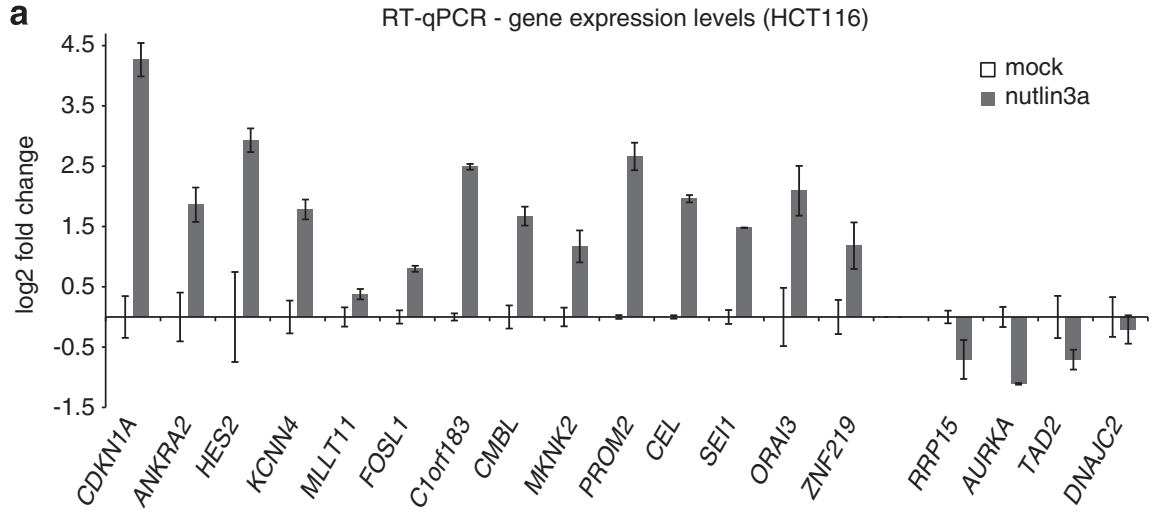

b

b

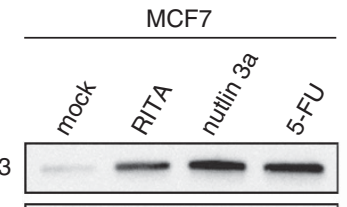

aurora a

actin

C

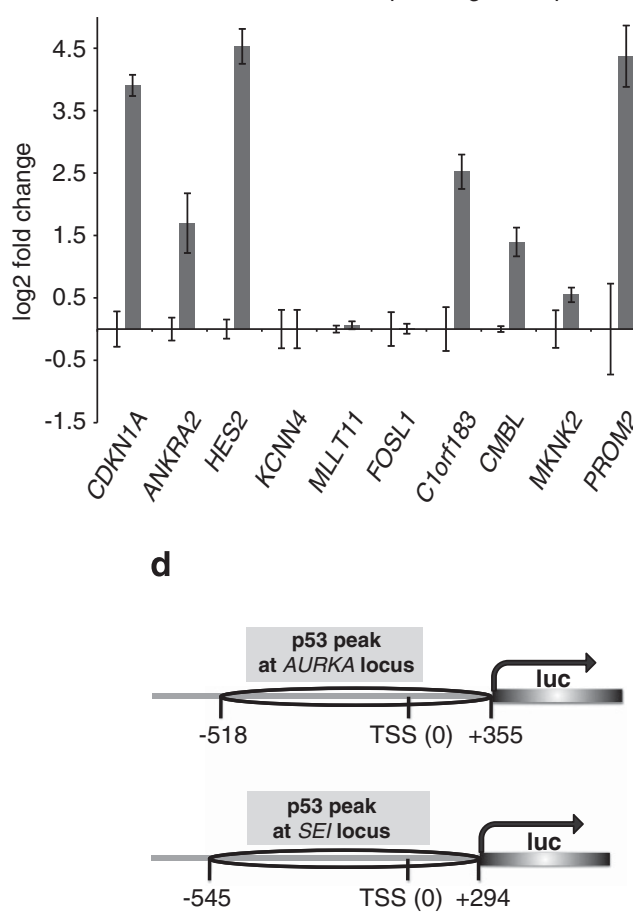

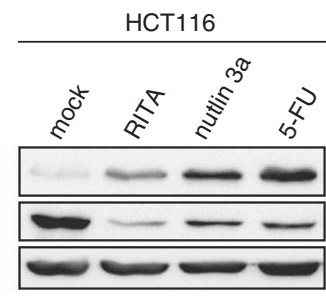

RT-qPCR - gene expression levels (HCT116 p53-/-) $\square$ mock

p53 
Table 2 Transcription factors predicted to have their binding sites in the vicinity ( $\pm 100 \mathrm{bp})$ of the p53-binding sites

\begin{tabular}{|c|c|c|c|c|c|c|c|c|c|c|c|}
\hline \multicolumn{4}{|c|}{ Commmon for induced and repressed } & \multicolumn{4}{|c|}{ Unique for induced } & \multicolumn{4}{|c|}{ Unique for Repressed } \\
\hline Factor & $\begin{array}{c}\text { N sites } \\
\text { per } 1 \text { kbp }\end{array}$ & Enrich & $P$-value & Factor & $\begin{array}{c}\mathrm{N} \text { sites } \\
\text { per } 1 \mathrm{kbp}\end{array}$ & Enrich & $P$-value & Factor & $\begin{array}{c}\text { N sites } \\
\text { per } 1 \text { kbp }\end{array}$ & Enrich & $P$-value \\
\hline $\mathrm{AHR}$ & 0.7117 & 1.8792 & 0.001829 & BACH2 & 0.2491 & 2.587 & 0.0098112 & ARNT & 1.7143 & 2.811 & 3.59E-05 \\
\hline AP2 & 5.1246 & 3.1678 & $9.43 E-40$ & ETV7 & 2.9181 & 1.3733 & 0.0006481 & BCL6 & 2.0714 & 1.9207 & 0.0017116 \\
\hline AP4 & 1.2278 & 1.6777 & 0.0006008 & HAND1 & 4.1815 & 1.494 & 7.40E-07 & $\overline{\text { DEAF1 }}$ & 0.2143 & 33.38 & 0.0021042 \\
\hline$A R$ & 0.3559 & 2.1322 & 0.0095111 & HNF1A & 0.1779 & 3.9597 & 0.0049341 & $\mathrm{HIF} 1 \mathrm{~A}$ & 1.2143 & 2.6642 & 0.0007813 \\
\hline CP2 & 4.7865 & 1.6139 & $7.10 \mathrm{E}-10$ & AP1 & 0.4804 & 2.5806 & 0.0004129 & & & & \\
\hline CREB/ATF & 1.9217 & 1.7404 & 7.09E-06 & MAF & 0.1779 & 6.9295 & 0.0005729 & & & & \\
\hline E2F & 1.2278 & 3.4153 & $1.15 \mathrm{E}-11$ & MAZ & 0.7117 & 2.174 & 0.0002464 & & & & \\
\hline EBF & 0.9431 & 2.2258 & 1.73E-05 & MYOG & 5.8541 & 1.416 & 2.89E-07 & & & & \\
\hline EGR1 & 0.8363 & 3.7221 & 3.98E-09 & NFKB & 1.121 & 1.7289 & 0.0005755 & & & & \\
\hline ETS1 & 1.3701 & 2.1558 & 6.09E-07 & NR3C1 & 0.1068 & 16.631 & 0.0018787 & & & & \\
\hline HIC1 & 0.4804 & 3.7419 & $7.46 \mathrm{E}-06$ & PAX6 & 8.2206 & 1.177 & 0.0020025 & & & & \\
\hline NR5A2 & 1.1744 & 1.5373 & 0.0037999 & SMAD3 & 4.6085 & 1.2729 & 0.0008693 & & & & \\
\hline PAX3 & 5.8363 & 1.2877 & 0.0001103 & SP3 & 4.2705 & 1.974 & $3.22 E-15$ & & & & \\
\hline PAX4 & 1.2811 & 1.5234 & 0.0030804 & ZВТВ33 & 0.2491 & 2.985 & 0.0044078 & & & & \\
\hline PAX5 & 6.9751 & 1.6266 & 5.52E-14 & & & & & & & & \\
\hline PPARA & 6.4057 & 1.2319 & 0.000642 & & & & & & & & \\
\hline SP1 & 1.1566 & 5.4596 & $2.06 \mathrm{E}-16$ & & & & & & & & \\
\hline STAT & 2.2143 & 1.8347 & 0.0022804 & & & & & & & & \\
\hline TEAD-2 & 1.2989 & 3.0658 & 7.00E-11 & & & & & & & & \\
\hline TP53 & 3.2562 & 8.0514 & 4.66E-55 & & & & & & & & \\
\hline V-MYB & 0.8541 & 2.2174 & 4.48E-05 & & & & & & & & \\
\hline VDR & 4.7687 & 1.6693 & $6.62 E-11$ & & & & & & & & \\
\hline WT1 & 7.0463 & 2.3304 & 8.54E-34 & & & & & & & & \\
\hline ZBTB7A & 10.854 & 1.6118 & $3.70 \mathrm{E}-20$ & & & & & & & & \\
\hline ZF5 & 7.8114 & 3.3521 & $6.24 \mathrm{E}-64$ & & & & & & & & \\
\hline ZFP67 & 0.7651 & 11.919 & $1.58 \mathrm{E}-16$ & & & & & & & & \\
\hline ZIC3 & 7.8114 & 1.4215 & 2.58E-09 & & & & & & & & \\
\hline
\end{tabular}

Expression level after nutlin3a treatment: underlined - induced; bold - repressed; plain - no changes.

suggesting that changes in their expression might in turn modulate the regulation of p53 targets.

Sp1 as a key determinant of alternative transcriptional program induced by RITA. Sp1 is one of the top factors whose RE are enriched in p53-bound sites (Table 2). Taken together with our recent finding that Sp1 contributes to the induction of apoptosis by RITA in MCF7 cells, ${ }^{26}$ this prompted us to test whether Sp1 might be one of the factors that confer specificity to RITA-induced transcriptional response.

Therefore, we performed microarray analysis in MCF7 cells stably depleted for Sp1 by short hairpin RNA, treated or not treated with RITA. We studied the expression of a set of 51 genes bound by p53 both upon nutlin3a and RITA treatment, but whose expression was significantly changed only by nutlin3a, but not RITA.

Strikingly, the expression of genes induced by RITA in Sp1depleted cells was very similar to that in nutlin3a-treated cells; these data sets were clustered together (Figure 5a). Thus, Sp1 depletion converted RITA-induced expression profile of these genes to the nutlin3a-like pattern.

Analysis of genes induced by RITA in Sp1-depleted cells revealed the robust activation of expression of negative p53 regulators MDM2 and Notch (Figure 5a). Induction of these two negative feedback loops can attenuate p53-iduced biological response by RITA in Sp-1 depleted cells, as it has been shown that enhanced expression of Mdm2 and Notch by nutlin3a restrains the potential therapeutic efficacy of nutlin3a. ${ }^{27,28}$ Further, the expression of pro-proliferative genes ALDH1A13, EDN2, Plk2 and FOSL1 was strongly induced, which might also counteract growth suppression. In addition, two pro-apoptotic genes $B B C 3$ and $E D A 2 R$ were also induced to the same level as upon nutlin3a treatment. However, these genes are not expected to contribute to nutlin3a-induced outcome, because it is mainly growth arrest/ senescence in these cells.

Thus, the induction of two negative feedback loops and several pro-proliferative genes upon p53 activation by RITA in the absence of Sp1 might play a role in attenuation of apoptosis, which we observe in RITA-treated Sp1-depleted cells $^{26}$ (Figure 5b, upper panel). Sp1 silencing did not affect nutlin3a-induced suppression of MCF7 cells (Figure 5b, lower panel), suggesting that $\mathrm{Sp} 1$ does not play a role in nutlin3ainduced p53 response.

Taken together, our findings implicate Sp1 as one of the key determinants of alternative transcriptional programs elicited by p53 upon RITA and nutlin3a treatment. Because the set of differentially expressed genes, which we analyzed, was bound by p53 upon both treatments, these results imply that Sp1 has a discriminatory impact on some p53-target genes after RITA treatment.

Antagonistic interaction between p53 and STAT3 at p53-repressed promoters. As shown in Table 1, p53-STAT composite element is enriched in p53-bound and repressed genes. Because STAT3 is an important oncogene and a promising target for anticancer therapy, we studied its impact on p53 targets. 


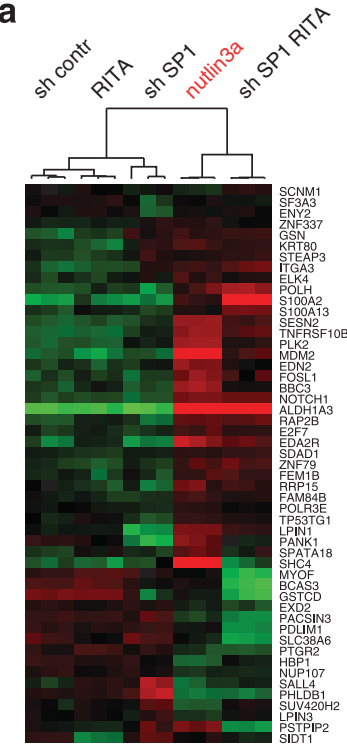

C

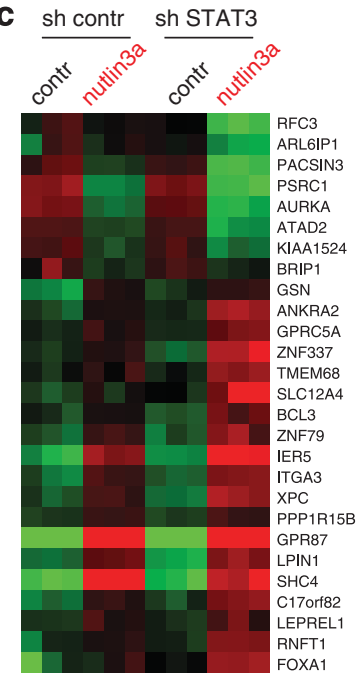

e

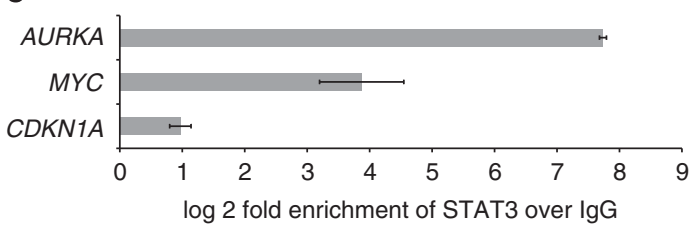

b
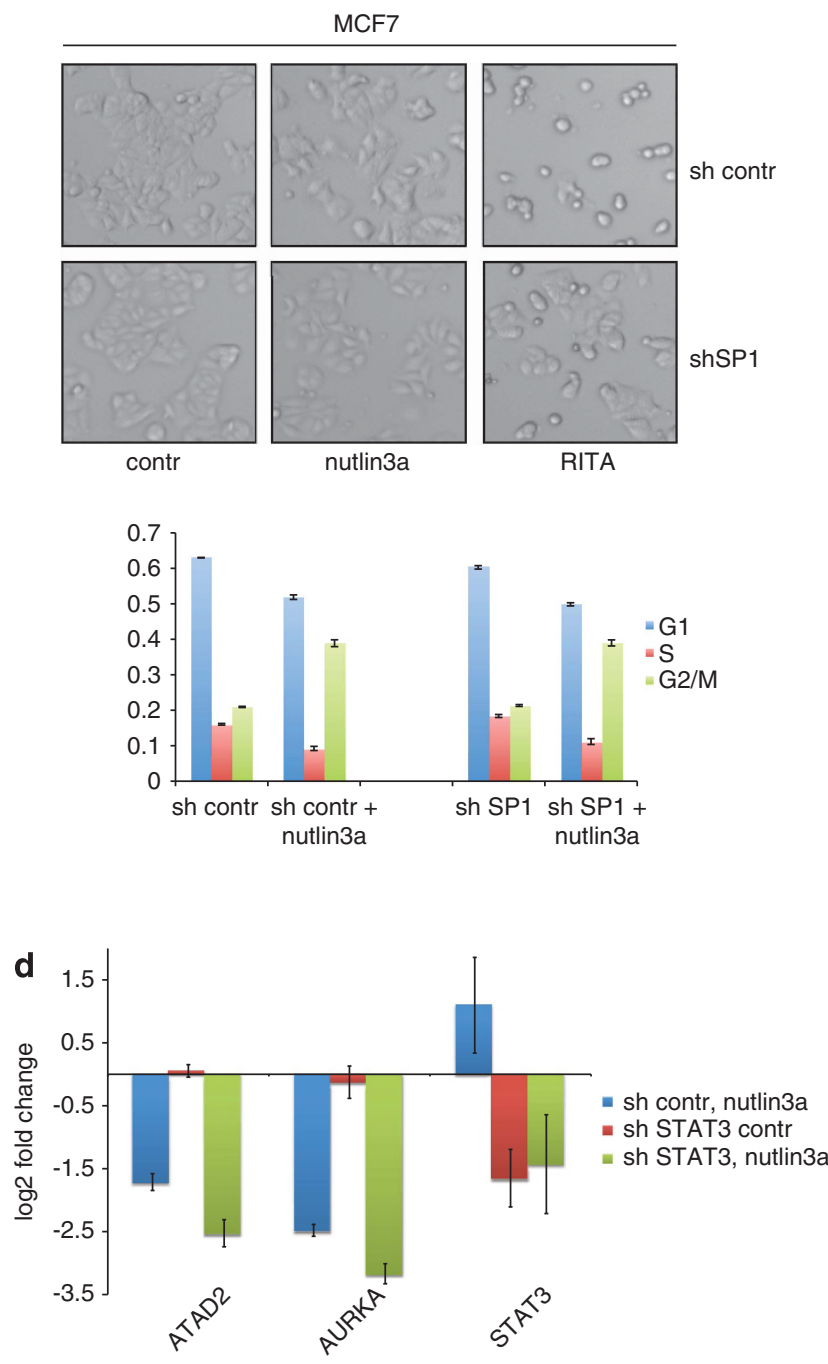

f

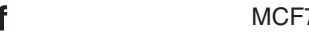

contr
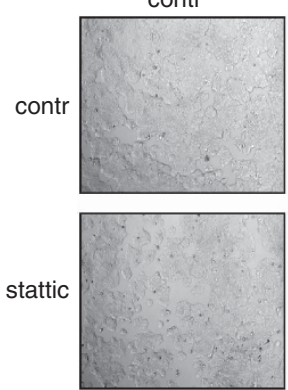

MCF7 nutlin3a
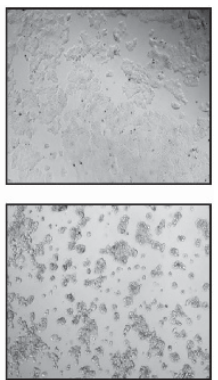

Figure 5 Identification of Sp1 and STAT3 as important modulators of p53 transcriptional activity. (a) The heatmap shows the results of gene expression analysis of RITAtreated MCF7 cells in which Sp1 was stably depleted by short hairpin RNA (shRNA). (b) Sp1 depletion attenuated RITA-induced apoptosis, but did not affect nutlin3a-induced growth arrest, as assessed by microscopy analysis (upper panel) and FACS of PI-stained cells (lower panel). (c) The heatmap shows the results of gene expression analysis of nutlin3a-treated MCF7 cells in which STAT3 was stably depleted by means of shRNA. (d) Depletion of STAT3 by shRNA enhances p53-mediated repression of AURKA and ATAD2 as assessed by GPCR in MCF7 cells treated with nutlin3a. (e) STAT3 ChIP in MCF7 cells demonstrated the binding of STAT3 to the promoter of AURKA. CDKN1A served as a negative and MYC as a positive control for STAT3 chromatin binding. (f) Inhibition of STAT3 by stattic facilitated the growth-suppression effect of nutlin3a as assessed by light microscopy analysis of the MCF7 after $48 \mathrm{~h}$ of treatment. See also Supplementary Figure S5 
Analysis of gene expression profiles upon p53 activation by nutlin3a in STAT3-depleted MCF7 cells showed that STAT3 silencing promotes p53 transcriptional activity, as a large set of p53 targets was affected by nutlin3a to a greater extent in STAT3-depleted cells (Figure 5c). Notably, AURKA and ATAD2, which we identified and validated as direct p53 targets, are also among STAT3-regulated genes (Figure $5 \mathrm{c}$ ). Indeed, qPCR analysis confirmed that their repression by p53 is stronger upon STAT3 depletion (Figure $5 \mathrm{~d}$ ), suggesting that STAT3 hampers p53-mediated repression of AURKA and ATAD2.

Furthermore, we found a significant binding of STAT3 to the AURKA promoter (Figure 5e), suggesting that STAT3 antagonizes p53-mediated repression of AURKA by direct binding to the promoter.

Next, we found that the growth suppression by nutlin3a was facilitated upon chemical inhibition of STAT3 by stattic (STAT three inhibitory compound), as well as by knockdown of STAT3 (Figure $5 f$ and Supplementary Figure S6, respectively), suggesting that STAT3 counteracts p53-induced biological response. Taken together, our results suggest that p53 reactivation by small molecules combined with STAT3 inhibition might have a beneficial effect in the clinical setting.

Moreover, we found that the direct p53 target genes $A U R K A$ and ATAD2 were among the most significantly differentially expressed genes in mutant compared with wildtype p53 breast tumor samples (Supplementary Figure S7), suggesting their high importance in p53-mediated prevention of tumor progression in patients.

\section{Discussion}

Our data suggest that the most frequently occupied p53 sites were the same upon p53 induction by three different stimuli, nutlin3a, RITA or chemotherapeutic drug 5-FU. Thus, this pattern can be thought of as a 'default p53 program'. This is in line with the conclusions of recent ChIP-chip and ChIP-seq studies that the occupancy of the core p53-binding sites is similar regardless of the mechanism of p53 activation and cell fate. ${ }^{29,30}$ Among the 'p53 default program' genes, we identified 61 known p53-target genes that are bound by p53 independently of the cell context and mechanism of p53 activation.

Parallel analysis of p53 genomic occupancy and gene expression allowed to identify 280 novel p53 target genes. Interestingly, the promoters of 63 out of 280 genes have been previously found to be bound by p53 in U2OS cells upon actinomycin $D$ treatment using ChIP-chip analysis ${ }^{11}$ (Supplementary Table S10, marked with a star).

Only $41 \%$ of genes bound by p53 were differently expressed upon nultin3a. Thus, the binding of $\mathrm{p} 53$ per se is not sufficient to regulate gene expression. Recent study suggests that although p53 phosphorylation at S15 and S46 does not significantly affect the pattern of p53 binding to promoters, it appears to affect p53 transcriptional program, ${ }^{30}$ probably via recruitment of additional factors.

Our study highlights the key role of TFs bound to p53regulated promoters in the selective regulation of target genes by $\mathrm{p} 53$. We show that $\mathrm{Sp} 1$ can redirect $\mathrm{p} 53$ towards a specific transcriptional program facilitating the induction of apoptosis.
Sp1 modulates p53 transcriptional program via attenuation of the p53-mediated induction of two negative feedback loops and pro-proliferative genes, as well as via cooperation with p53-mediated repression of metabolic genes, as we previously showed. ${ }^{26}$

Compared with gene activation, less is known about p53mediated transcriptional repression. ${ }^{18}$ Our analysis of novel p53 target genes implies that the transrepression by p53 might be achieved due to the direct binding to its consensus only in certain cases. It is possible that p53-mediated repression might be indirect and/or the binding mode of p53 is different (i.e., via another TF or non-consensus binding site). Another possibility is that p53 regulates these genes via 'looping mechanism'. 31

The analysis of composite binding motifs revealed the enrichment of E2F-p53 and IRF-p53 composite sites, suggesting the involvement of these two factors in p53mediated repression of genes on a larger scale than previously anticipated. Moreover, our data suggest that another mode of p53 repression involves inhibition of protooncogenic TF, such as STAT3, MYB and others, via direct binding to promoters in the close proximity to their RE.

We found that STAT3, important oncogene and a promising target for anticancer therapy, antagonizes p53 activity at several promoters, including the newly identified p53 target AURKA. This implies that the combination of p53 activators with STAT3 inhibitors might be beneficial in a clinic.

Low occupancy of p53 at repressed promoters most probably reflects lower number of cells in which p53 is bound to these promoters at a given moment, that is, more transient binding mode. Transient binding of p53 to repressed promoters might be related to a different mode of transcription activation versus repression. Activation of transcription requires the constant presence of an activator to ensure open chromatin structure and accessibility for transcriptional machinery. In contrast, once the repressive chromatin state at the promoter is established, it is self-maintained in the absence of an inducing signal due to initial modification, providing new binding sites for the enzymes that maintain chromatin modifications. Therefore, it does not require the continuous presence of the initial repressor. ${ }^{32}$

In conclusion, our data strongly suggest that p53-mediated target gene expression upon activation by nutlin3a, RITA or $5-\mathrm{FU}$ is determined not only by a selective binding of p53 to its target promoters but also depends on cooperating or antagonizing TFs. These results are expected to help in a future to harness p53 activity by targeting p53 modulators and achieve a desired p53 transcriptional response.

\section{Materials and Methods}

Cell-based assays. MCF7 is a human breast carcinoma line and HCT116 and HCT116 p53 - I - cells, a gift from B Vogelstein, are colon carcinoma. Cell lines were treated with $1 \mu \mathrm{M}$ RITA, $10 \mu \mathrm{M}$ nutlin3a or $100 \mu \mathrm{M} \mathrm{5-FU}$ for 8 or $4 \mathrm{~h}$ before analysis. Stattic $5 \mu \mathrm{M}$ was used to inhibit STAT3 activity. The pGl3 luciferase construct, used for the cloning of promoter fragments, was provided by $S$ Mkrtchian of the Karolinska Institutet. The AURKA promoter $(-518$ to +355$)$ and SEl1 promoter $(-545$ to +294$)$ were cloned using the primers listed in Supplementary Table S11. Sp1-depleted cell line was established as described in Zawacka-Pankau et al. ${ }^{26}$ Transfections were performed using Lipofectamine 2000 (Invitrogen, Grand Island, NY, USA). Luciferase activity was measured using the Dual-Luciferase Reporter System (Promega, Madison, WI, USA) and analyzed as described before. ${ }^{33}$ 
Cell viability and fluorescence-activated cell sorting analysis were performed as previously described. ${ }^{15}$ Western blotting was performed according to the standard procedures. The proteins were detected using the following antibodies: anti-p53 DO-1 (Santa Cruz Biotechnology, Santa Cruz, CA, USA), anti-Aurora A ab13824 (Abcam, Cambridge, UK) and anti-beta-actin (Sigma-Aldrich, St. Louis, MO, USA).

ChIP and ChIP-seq experiment. ChIP in MCF7 cells, library preparation, massive parallel sequencing and ChIP-Seq primary analysis was performed as previously published. ${ }^{34}$ Sc-126 (Santa-Cruz Biotechnology, Santa Cruz, CA, USA), Genespin DO-1 p53 and Sc-2025 mouse IgG antibodies were used for IP.

Approximately 6 million high-quality sequencing reads were mapped to the human genome (NCBI36) and used to calculate the height of the peaks as the number of overlapping reads extended to the fragment length. The number of extended reads in each p53 peak was compared with the number of extended reads in the same region in an IgG-precipitated sample and enrichments were calculated. Data are publicly available from the NCBI sequence Read Archive and in Supplementary data (Supplementary Tables S1-S4). Data are archived at NCBI Sequence Read Archive under Accession SRP007261.

Intersection of the peaks $(P<0.05)$ after different treatments was performed with a web-based tool, Galaxy, ${ }^{16}$ and imported into R (http://www.r-project.org/). Venn diagrams were plotted with Vennerable R package (http://R-Forge.R-project.org/ projects/vennerable/). The distribution of the peaks around the TSS was calculated using the ChIPpeakAnno package. ${ }^{35}$ For de novo motif discovery analysis, we used the $\mathrm{MEME}^{36}$ or $\mathrm{p} 53 \mathrm{MH}^{37}$ and $\mathrm{p} 53 \mathrm{~S} \mathrm{can}^{11}$ programs. As input data, we used sequences at a distance $\pm 200 \mathrm{bp}$ from the position of the peak maximum height.

The presence of TF-binding sites located at $\pm 100 \mathrm{bp}$ from p53-binding sites in ChIP-Seq fragments and composite patterns were analyzed with the tool MATCH that uses a library of about 600 positional weight matrices (PWMs) from the TRANSFAC database for vertebrate TFs. ${ }^{38}$ Overrepresentation of TF-binding sites and composite elements (TF site pairs) were computed by the F-Match algorithm and composite module analyst program (Composite Module Analysts) that is implemented in the geneXplain platform (www.genexplain.com).

Microarray experiment. Expression profiling of MCF7 cells was performed using Affymetrix (Santa Clara, CA, USA) human genome 219 arrays as previously described. ${ }^{26}$ Primer sequences for qPCR are listed in Supplementary Table S13.

\section{Conflict of Interest}

The authors declare no conflict of interest.

Acknowledgements. This study was funded by the Swedish Cancer Society, the Swedish Research Council, the Cancer Society of Stockholm, Karolinska Institutet (ACT! Temacentrum), Ragnar Söderberg's Foundation, and Robert Lundberg's memorial foundation. This work was also supported by European Commission FP6 and FP7 (SysCol), the Russian federal program 'Living systems', State Contract no. 11.519.11.2031 and by BMBF project 'GerontoShield'. The publication reflects only the authors' views. The European Commission is not liable for any use of information herein. We are greatly indebted to all of our colleagues who shared valuable reagents and cell lines with us. We are thankful to Diana Grochová, Andreas Warnecke and Martin Enge for fruitful discussions and comments during the manuscript preparation.

1. Goh AM, Coffill CR, Lane DP. The role of mutant p53 in human cancer. J Pathol 2011; 223 116-126.

2. Vousden KH, Prives C. Blinded by the light: the growing complexity of p53. Cell 2009; 137 413-431.

3. Vassilev LT, Vu BT, Graves B, Carvajal D, Podlaski F, Filipovic Z et al. In vivo activation of the p53 pathway by small-molecule antagonists of MDM2. Science 2004; 303: 844-848.

4. Issaeva N, Bozko P, Enge M, Protopopova M, Verhoef LG, Masucci M et al. Small molecule RITA binds to p53, blocks p53-HDM-2 interaction and activates p53 function in tumors. Nat Med 2004; 10: 1321-1328.

5. Ding K, Lu Y, Nikolovska-Coleska Z, Wang G, Qiu S, Shangary S et al. Structure-based design of spiro-oxindoles as potent, specific small-molecule inhibitors of the MDM2-p53 interaction. J Med Chem 2006; 49: 3432-3435.

6. Bykov VJ, Issaeva N, Shilov A, Hultcrantz M, Pugacheva E, Chumakov P et al. Restoration of the tumor suppressor function to mutant p53 by a low-molecular-weight compound. Nat Med 2002; 8: 282-288.
7. Lain S, Hollick JJ, Campbell J, Staples OD, Higgins M, Aoubala M et al. Discovery, in vivo activity, and mechanism of action of a small-molecule p53 activator. Cancer Cell 2008; 13: 454-463.

8. Enge M, Bao W, Hedstrom E, Jackson SP, Moumen A, Selivanova G. MDM2-dependent downregulation of $\mathrm{p} 21$ and hnRNP $\mathrm{K}$ provides a switch between apoptosis and growth arrest induced by pharmacologically activated p53. Cancer Cell 2009; 15: 171-183.

9. el-Deiry WS, Kern SE, Pietenpol JA, Kinzler KW, Vogelstein B. Definition of a consensus binding site for p53. Nat Genet 1992; 1: 45-49.

10. Vousden KH, Lu X. Live or let die: the cell's response to p53. Nat Rev Cancer 2002; 2 : 594-604.

11. Smeenk L, van Heeringen SJ, Koeppel M, van Driel MA, Bartels SJ, Akkers RC et al. Characterization of genome-wide p53-binding sites upon stress response. Nucleic Acids Res 2008; 36: 3639-3654.

12. Wei $\mathrm{CL}$, Wu $Q$, Vega VB, Chiu KP, Ng P, Zhang T et al. A global map of $p 53$ transcriptionfactor binding sites in the human genome. Cell 2006; 124: 207-219

13. Dell'Orso S, Fontemaggi G, Stambolsky P, Goeman F, Voellenkle C, Levrero M et al. ChIP-on-chip analysis of in vivo mutant p53 binding to selected gene promoters. OMICS 2011; 15: 305-312.

14. Sampath D, Rao VA, Plunkett W. Mechanisms of apoptosis induction by nucleoside analogs. Oncogene 2003; 22: 9063-9074.

15. Grinkevich VV, Nikulenkov F, Shi Y, Enge M, Bao W, Maljukova A et al. Ablation of key oncogenic pathways by RITA-reactivated p53 is required for efficient apoptosis. Cancer Cell 2009; 15: 441-453.

16. Blankenberg D, Von Kuster G, Coraor N, Ananda G, Lazarus R, Mangan M et al. Galaxy: a web-based genome analysis tool for experimentalists. Curr Protoc Mol Biol 2010; Unit 19.10: 1-21

17. Menendez D, Inga A, Resnick MA. The expanding universe of $p 53$ targets. Nat Rev Cancer 2009; 9: 724-737.

18. Riley $\mathrm{T}$, Sontag $\mathrm{E}$, Chen $\mathrm{P}$, Levine A. Transcriptional control of human p53-regulated genes. Nat Rev Mol Cell Biol 2008; 9: 402-412.

19. Kel A, Konovalova T, Waleev T, Cheremushkin E, Kel-Margoulis O, Wingender E. Composite module analyst: a fitness-based tool for identification of transcription factor binding site combinations. Bioinformatics 2006; 22: 1190-1197.

20. Polager S, Ginsberg D. p53 and E2f: partners in life and death. Nat Rev Cancer 2009; 9: 738-748.

21. Kitagawa M, Aonuma M, Lee SH, Fukutake S, McCormick F. E2F-1 transcriptional activity is a critical determinant of Mdm2 antagonist-induced apoptosis in human tumor cell lines. Oncogene 2008; 27: 5303-5314.

22. Lee SH, Kim JW, Lee HW, Cho YS, Oh SH, Kim YJ et al. Interferon regulatory factor-1 (IRF-1) is a mediator for interferon-gamma induced attenuation of telomerase activity and human telomerase reverse transcriptase (hTERT) expression. Oncogene 2003; 22: 381-391.

23. Shi L, Ko S, Kim S, Echchgadda I, Oh TS, Song CS et al. Loss of androgen receptor in aging and oxidative stress through Myb protooncoprotein-regulated reciprocal chromatin dynamics of p53 and poly(ADP-ribose) polymerase PARP-1. J Biol Chem 2008; 283: 36474-36485

24. Nguyen TT, Cho K, Stratton SA, Barton MC. Transcription factor interactions and chromatin modifications associated with p53-mediated, developmental repression of the alpha-fetoprotein gene. Mol Cell Biol 2005; 25: 2147-2157.

25. Naruganahalli KS, Lakshmanan M, Dastidar SG, Ray A. Therapeutic potential of Aurora kinase inhibitors in cancer. Curr Opin Investig Drugs 2006; 7: 1044-1051.

26. Zawacka-Pankau J, Grinkevich VV, Hunten S, Nikulenkov F, Gluch A, Li H et al. Inhibition of glycolytic enzymes mediated by pharmacologically activated p53: targeting Warburg effect to fight cancer. J Biol Chem 2011; 286: 41600-41615.

27. van Leeuwen IM, Higgins $M$, Campbell J, Brown CJ, McCarthy AR, Pirrie $L$ et al. Mechanism-specific signatures for small-molecule p53 activators. Cell Cycle 2011; 10: $1590-1598$

28. Secchiero $\mathrm{P}$, Melloni $\mathrm{E}$, di lasio MG, Tiribelli M, Rimondi $\mathrm{E}$, Corallini $\mathrm{F}$ et al. Nutlin-3 up-regulates the expression of Notch1 in both myeloid and lymphoid leukemic cells, as part of a negative feedback antiapoptotic mechanism. Blood 2009; 113: 4300-4308.

29. Shaked H, Shiff I, Kott-Gutkowski M, Siegfried Z, Haupt Y, Simon I. Chromatin immunoprecipitation-on-chip reveals stress-dependent p53 occupancy in primary normal cells but not in established cell lines. Cancer Res 2008; 68: 9671-9677.

30. Smeenk L, van Heeringen SJ, Koeppel M, Gilbert B, Janssen-Megens E, Stunnenberg HG et al. Role of p53 serine 46 in p53 target gene regulation. PLoS One 2011; 6: e17574.

31. Jackson P, Mastrangelo I, Reed M, Tegtmeyer P, Yardley G, Barrett J. Synergistic transcriptional activation of the MCK promoter by p53: tetramers link separated DNA response elements by DNA looping. Oncogene 1998; 16: 283-292.

32. Ptashne M. Principles of a switch. Nat Chem Biol 2011; 7: 484-487.

33. Wang B, Xiao Z, Ren EC. Redefining the p53 response element. Proc Natl Acad Sci USA 2009; 106: 14373-14378.

34. Wei GH, Badis G, Berger MF, Kivioja T, Palin K, Enge M et al. Genome-wide analysis of ETS-family DNA-binding in vitro and in vivo. EMBO J 2010; 29: 2147-2160.

35. Zhu LJ, Gazin C, Lawson ND, Pages H, Lin SM, Lapointe DS et al. ChIPpeakAnno: a bioconductor package to annotate ChIP-seq and ChIP-chip data. BMC Bioinformatics 11: 237

36. Bailey TL, Elkan C. Fitting a mixture model by expectation maximization to discover motifs in biopolymers. Proc Int Conf Intell Syst Mol Biol 1994; 2: 28-36. 
37. Hoh J, Jin S, Parrado T, Edington J, Levine AJ, Ott J. The p53MH algorithm and its application in detecting p53-responsive genes. Proc Natt Acad Sci USA 2002; 99: 8467-8472.

38. Kel AE, Gossling E, Reuter I, Cheremushkin E, Kel-Margoulis OV, Wingender E. MATCH: a tool for searching transcription factor binding sites in DNA sequences. Nucleic Acids Res 2003; 31: 3576-3579
This work is licensed under the Creative Commons

Attribution-NonCommercial-No Derivative Works 3.0
Unported License. To view a copy of this license, visit http:// creativecommons.org/licenses/by-nc-nd/3.0/

Supplementary Information accompanies the paper on Cell Death and Differentiation website (http://www.nature.com/cdd) 